ARTICULO DE REVISIÓN BIBLIOGRÁFICA

\title{
La relación de confianza y compromiso en la intención de compra
}

\section{Trust and commitment relationship in the purchase intention}

\author{
Ángel Leonardo Ávila Castañeda ${ }^{1}$ \\ Rosa Alexandra Chaparro Guevara ${ }^{2}$
}

1. Profesional de mercadeo. Maestría en Dirección Estratégica de Marketing. Universidad Agustiniana. Bogotá. https://orcid.org/0000-0001-9988-3066

2. Administradora de empresas. Maestría en Gestión de la Innovación. Universidad Agustiniana. Bogotá. https://orcid.org/0000-0002-6238-9432

\section{RESUMEN}

Este estudio tuvo como objetivo identificar la relación sobre la confianza y el compromiso en la intención de compra, específicamente en el campo del marketing, evaluando a profundidad los principales temas y métodos desarrollados. A partir de artículos establecidos en diferentes bases de datos, se llevó a cabo una revisión sistemática de la literatura, identificando 169 artículos relacionados con las variables establecidas; asimismo, se analizaron a profundidad 39 artículos tomados como referencia para el desarrollo de la revisión.

Se identificó que el tema se estudia en varios entornos: como en el consumo de alimentos saludables; los productos certificados o sellos de garantía; en las acciones de responsabilidad social que desarrollan las empresas; las condiciones económicas; en el entorno digital; en el país de origen y los anuncios en redes sociales, entre otros. Este trabajo también identificó que la mayor parte de la investigaciones se encuentra indexada en revistas enfocadas en marketing, sostenibilidad, negocios, responsabilidad social, análisis económico, alimentos y comportamiento del consumidor. 
COMPÁS EMPRESARIAL No 32

Vol. 11 - 1ER SEMESTRE 2021

ISSN: 2075-8960

Universidad Privada del Valle - Bolivia

https://doi.org/10.52428.20758960.v11i32.62

Es decir, este trabajo contribuye a consolidar la investigación académica del concepto de la intención de compra y las diferentes variables y metodológicas que se relacionan, demostrando que la confianza y el compromiso genera una fuerte relación en el proceso de decisión e intención de compra de los consumidores.

Palabras claves: Confianza. Compromiso e intención de compra. Consumidor.

\begin{abstract}
This study aimed to identify the trust and commitment to purchase intention, specifically in the marketing field, deep evaluation of the main topics and methods develop. Based on articles established in different databases, a systematic literature review was carried out, identifying 169 articles related to established variables; likewise, 39 articles were deeply analyzed, taken as a reference for the review development.
\end{abstract}

It was identified that the subject is studied in various settings: as in the healthy food consumption; certified products or guarantee seals; in the social responsibility actions carried out by companies; economic conditions; in the digital environment; in the origin country and advertisement on social networks, among others. This work also identified that most of the research is indexed in magazines focused on marketing, sustainability, economic analysis, food, and consumer behavior.

Therefore, this paper contributes to consolidate the academic research on the concept of purchase intention and the different variables and methodologies that are related, demonstrating that trust and commitment generate a strong relationship in the decision process and the consumers' purchase intention.

Keywords: Commitment and purchase intention. Consumer. Trust. 
Vol. 11 - 1ER SEMESTRE 2021

ISSN: 2075-8960

Universidad Privada del Valle - Bolivia

https://doi.org/10.52428.20758960.v11i32.62

\section{INTRODUCCIÓN}

Dentro de un entorno hipercompetitivo, la confianza y compromiso de los consumidores hacia las marcas cada vez está más fracturado, ya que se enfrentan a ofertas que no trasmiten ventajas competitivas claras y llamativas; en muchos casos, por el afán de las marcas de conservar sus cuotas de mercado, se desarrollan acciones de corto plazo sin entender muy bien la intención y el comportamiento de los consumidores en un futuro, siendo estos factores clave para establecer el pronóstico de la demanda.

El concepto intención de compra ha representado para las marcas y la academia un gran reto en sus propósitos de estudio. Por un lado, permitiendo generar un gran aporte conceptual y logrando establecerla como una proyección del comportamiento del consumidor en su proceso de decisión de compra Torres \& Padilla (2013); y por otro, establecen los métodos o métricas para poder identificar esos comportamientos, tal como lo desarrollaron Calvo-Porral et al., (2013) en su estudio comparan dos modelos de ecuaciones estructurales aplicados a la intención de compra.

La intención de compra puede verse afectada desde variables como el producto, precio canal de distribución o promoción; o desde un enfoque subjetivo como la experiencia, conocimiento, percepción, o conocimiento de una marca; el interés de los autores está en identificar desde una investigación teórico-descriptiva de tipo documental, los principales aportes de cada uno de los criterios de búsqueda, tales como confianza, compromiso e intención de compra para lo cual se establecen etapas para el estudio las cuales parten desde la definición del tema, búsqueda bibliográfica, selección y análisis de los documentos para finalmente establecer las conclusiones.

El propósito de la presente investigación es proporcionar una visión general de diferentes estudios dentro de este campo, propiamente de los conceptos de confianza, compromiso e intención de compra. 
Vol. 11 - 1ER SEMESTRE 2021

ISSN: 2075-8960

Universidad Privada del Valle - Bolivia

https://doi.org/10.52428.20758960.v11i32.62

\section{DESARROLLO}

Para el desarrollo de la investigación documental se utilizaron varias fuentes documentales, estableciendo discernimientos de inclusión realizando su búsqueda en los meses de mayo a junio del 2020, utilizando descriptores de: Confianza del consumidor, compromiso del consumidor e intención de compra, en los cuales inicialmente se identifican 169 registros en diferentes áreas y escuelas de investigación obtenido en bases de datos como Web of Science, ScienceDirect y Google Academic, como se refleja en la Tabla 1.

\begin{tabular}{cc}
\hline Áreas de la investigación & Numero de registros \\
\hline Business Economics & 93 \\
Environmental sciences ecology & 14 \\
Communication & 11 \\
Environmental sciences ecology & 11 \\
Phychology & 10 \\
Science technology other topics & 8 \\
Information science library science & 6 \\
Food science technology & 6 \\
Health care sciences services & 5 \\
Sociology & 5 \\
\hline & \\
Fuente: Elaboración propia a partir de los resultados obtenidos de búsqueda en las bases de datos 2020.
\end{tabular}

Dentro de este proceso de búsqueda, se identifica los 10 principales títulos de fuentes donde se concentran los artículos en los cuales se destacan las áreas de marketing, negocios, sostenibilidad, economía y alimentos, como se presenta en la Tabla 2.

\begin{tabular}{c}
\hline Títulos de Fuentes \\
European journal of marketing \\
Cogent Business Management \\
Sustainability \\
Corporate social responsibility and environmental management \\
Economic annals xxi \\
Food control \\
Journal of marketing management \\
Journal of business research \\
Journal of interactive marketing \\
Journal of retailing and consumer services \\
Tabla 2 \\
Títulos de fuentes \\
Fuente: Elaboración propia a partir de los resultados obtenidos de búsqueda en las bases de datos 2020.
\end{tabular}


COMPÁS EMPRESARIAL No 32

Vol. 11 - 1ER SEMESTRE 2021

ISSN: $2075-8960$

Universidad Privada del Valle - Bolivia

https://doi.org/10.52428.20758960.v11i32.62

Como resultado de la búsqueda se logra identificar los constructos más pertinentes para el desarrollo del artículo de revisión, presentándose de la siguiente forma:

\section{a) Constructos de Confianza del consumidor}

El concepto de la confianza se convierte en una variable importante dentro del comportamiento humano y se analiza desde diferentes perspectivas, una de ellas desde lo emocional siendo un aspecto de relaciones cercanas como lo establecieron Larzelere \& Huston (1980), quienes instauraron la confianza como el resultado de la fiabilidad a las otras personas generando una respuesta emocional traducido en sentimientos o actitudes positivas.

Otra de las perspectivas de análisis en relación con la confianza es desde lo económico, siendo un indicador que intenta medir la impresión y expectativas que tienen los consumidores respecto a la situación económica del país y su hogar y su intención de compra para realizar gastos en bienes durables. Este indicador para la industria es de gran utilidad, ya que influye en la planificación de producción y en el desarrollo de sus acciones comerciales (Fedesarrollo, 2020).

Y desde la perspectiva del marketing, la confianza se considera como un factor clave en las relaciones entre las marcas y el consumidor y su influencia en el comportamiento a largo plazo afianzando la relación credibilidad, relevancia y fiabilidad logrando generar un vínculo de lealtad frente a la marca. (Flavian \& Guinalíu, 2006)

A partir de estos enfoques, se encuentran diferentes estudios en función de determinar el nivel de confianza; Ngo et al., (2020) examinan el papel de la confianza de los procesos de producción y de la marca en las verduras certificadas como seguras, confirmando que hay un efecto fuertemente directo en la confianza de los consumidores, convirtiéndose en un papel mediador entre la confianza de la cadena de abastecimiento y la confianza en la seguridad alimentaria. 
COMPÁS EMPRESARIAL No 32

Vol. 11 - 1ER SEMESTRE 2021

ISSN: 2075-8960

Universidad Privada del Valle - Bolivia

https://doi.org/10.52428.20758960.v11i32.62

Algo similar ocurre con Wang et al., (2020), quienes evaluaron la confianza de los consumidores chinos en la certificación de alimentos incorporando la variable de calidad percibida como un constructo en la teoría del comportamiento planificado, así como la información y procedencia de los organismos certificadores de los alimentos generando un importante impacto en la intención de compra de los consumidores.

Distinto de Zorio-Grima \& Merello (2020), que analizaron la relación de causalidad entre el índice de confianza del consumidor y el ecosistema de información económica, a partir de fuentes subjetivas como el sentimiento empresarial y objetivas, como la información económica de las empresas, estableciendo que el índice de confianza del consumidor es causal en las decisiones de consumo impactando en la situación económica de un país.

En cambio Jonkman et al., (2020) formaron la relación entre las noticias negativas y la confianza de los consumidores, confirmando su hipótesis de la dependencia de las dos variables y, especialmente, cuando las condiciones económicas están empeorando o el contexto del país no es favorable. De modo similar Boukes et al., (2019) demostraron que a medida que las personas estaba expuestas a noticias económicas relativamente positivas mejoraban las evaluaciones económicas nacionales y sus expectativas para él futuro.

La confianza también se ve afectada por sucesos que se hayan presentado años atrás, esta conclusión se ve reflejada en el estudio desarrollado por Li et al., (2019), donde encuentran que los consumidores que residen en el norte urbano de China todavía carecen de confianza en la seguridad de las fórmulas infantiles producidas en el país casi una década después del incidente de la leche contaminada con melamina en 2008.

En otros contextos, las empresas con propósitos sociales o sostenibles ejercen un factor motivacional en los consumidores, los valores sociales y ambientales son principales motivadores para la compra de productos, ya que brindan una confianza en la efectividad en la contribución social o ambiental, generando un beneficio personal convirtiéndose en un agente de cambio 
COMPÁS EMPRESARIAL No 32

Vol. 11 - 1ER SEMESTRE 2021

ISSN: 2075-8960

Universidad Privada del Valle - Bolivia

https://doi.org/10.52428.20758960.v11i32.62

disfrutando de productos saludables y de alta calidad, como se evidencia en el trabajo de (Bianchi et al., 2020)

Otro de los grandes retos para las empresas es identificar el nivel de confianza en el contexto digital, promoviendo la toma de decisiones de los consumidores con respecto a la interacción de la marca con el consumidor digital, exigiendo un mayor esfuerzo en ganarse la confianza de los consumidores, donde la taza de difusión y las cantidades de información son de gran importancia, realizando una integración de las tecnologías digitales y de comunicación (Popova et al., 2019).

Es así como Choi \& Lee, (2019) examinaron la influencia de la confianza en la información específica de las marcas de cosméticos en sus páginas web sobre la información específica de los productos y su relación con el valor percibido que identifican los consumidores, generando un efecto positivo en la intención de compra de esta categoría.

Para el sector de los seguros de vida, se establecen cuatro dimensiones, es como lo instituyeron Agyei et al., (2020) indicando que tanto el proveedor, el regulador, la situación económica y la información del prestador del servicio impactan significativamente en el nivel de confianza del consumidor.

\section{b) Constructos de Compromiso del Consumidor}

El propósito de la fidelización se da cuando las marcas son capaces de integrarse en la vida de los consumidores, es decir alcanzar una relación más profunda y cercana con el consumidor convirtiéndose en una apuesta de mediano y largo plazo, ya que esta se construye por medio de las acciones que realice la marca, partiendo del nivel de interés que genere para el consumidor, la facilidad de interacción y el nivel de confianza y satisfacción que la marca genera.

Es el caso de Bonillo, (2002) se forma el concepto bajo diferentes perspectivas: en primer lugar, desde un análisis actitudinal donde establece el compromiso desde un carácter afectivo y donde se incorporan emociones o sentimientos; el carácter cognitivo, englobando las percepciones, 
COMPÁS EMPRESARIAL No 32

Vol. 11 - 1ER SEMESTRE 2021

ISSN: $2075-8960$

Universidad Privada del Valle - Bolivia

https://doi.org/10.52428.20758960.v11i32.62

creencias y conocimientos; y el conductual, donde hace referencia al deseo, voluntad o intención. En segundo lugar, establece que el compromiso está relacionado con un comportamiento consistente en el tiempo; y finalmente, instaura que el compromiso debe estar presente en las dos partes es decir bajo un carácter reciproco.

Por otro lado, Garzaro et al., (2020) identifican el estado de la investigación sobre la interactividad y el compromiso, estableciendo que estos se estudian en varios entornos, como las páginas web, comunidades sociales, anuncios en redes sociales y telefonía móvil.

Es así como Naumann et al., (2020) midieron los efectos del compromiso de los clientes negativos junto con los clientes positivos, a través de dimensiones afectivas, cognitivas y comportamentales, generando una participación importante en el voz a voz que puedan generar los dos tipos de clientes. A su vez, Chen et al., (2020) propusieron un marco integrado del compromiso de los turistas con la autenticidad y conveniencia de la marca, creando un efecto directo en la intención de la recomendación, pero es bajo en el efecto de volver a visitar el lugar, a menos que identifique la reciprocidad o compromiso de la marca.

Por otro lado, Kaur et al. (2020) identificaron el efecto positivo de la integración a comunidades de marca por parte de los consumidores, generando interacciones sociales con otros miembros y proporcionando un entorno comunitario; así mismo, las marcas deben desarrollar ofertas personalizadas o estrategias de recompensa hedónica y utilitaria que permitan satisfacer las necesidades afectivas, cognitivas o sociales de los miembros de la comunidad, como recompensar a los que tengan una mayor participación o que actúen como influenciadores de la marca.

Para otros autores, el nivel de compromiso se ve afectado por los rasgos de personalidad y los valores personales, tal como lo relacionaron Marbach et al., (2019) quienes sugieren que la reflexión, apertura de experiencias el altruismo, como rasgos de personalidad, se correlacionan positivamente con el compromiso del consumidor y la conversación y automejora como valores personales moderan la relación entre estos tres rasgos de personalidad. 


\section{COMPÁS EMPRESARIAL No 32}

Vol. 11 - 1ER SEMESTRE 2021

ISSN: $2075-8960$

Universidad Privada del Valle - Bolivia

https://doi.org/10.52428.20758960.v11i32.62

Igualmente Dessart et al., (2019) comparten que el compromiso de las comunidades es un fenómeno importante pero se enfocan sobre la naturaleza del compromiso de las personas, estableciendo tres segmentos de consumidores comprometidos, el primero corresponde a los participantes emocionales, quienes confían en dimensiones afectivas dejándose llevar por las emociones generadas por la interacción con la marca. Los consumidores pensadores son el segundo segmento, quienes se caracterizan por su compromiso cognitivo a través de sus procesos mentales y finalmente los individuos activadores ya que para ellos el comportamiento activo de compartir, aprender y apoyar es la principal razón para desarrollar la lealtad.

En cambio para otros autores establecen el nivel de compromiso desde los diferentes impulsores o estímulos desarrollados por la marca, como lo es la página web, Connell et al., (2019), lograron identificar que las señales ambientales relacionadas con el producto impulsan y representan un punto de contacto vital para el desarrollo del compromiso del consumidor. Cabe destacar que para los consumidores también esperan una respuesta oportuna y pertinente por parte de la marca a través de sus medios digitales, es así como lo concluyo Sheng, (2019), donde afirma que la velocidad de respuesta por parte de la marca influye positivamente , así como Busalim et al., (2019) establecen que la calidad de los sitios web y la exactitud de la información proporcionan un impacto potencial en el compromiso de los clientes.

Por otra parte Shawky et al., (2019) reconocen el papel de las redes sociales como un elemento clave para la creación de comunidades comprometidas gracias a la comunicación bidireccional, sirviendo para las marcas como un canal extendido a los esfuerzos tradicionales de los medios de comunicación logrando llegar a grandes audiencias de manera económica construyendo relaciones e influenciando en los comportamientos de su público objetivo.

Finalmente, otros autores buscan establecer la relación del compromiso con el apego y el amor, Gómez, (2019) formó que el compromiso o participación es una activación del consumidor al momento de elegir y el apego se refleja en el afecto o amistad y que, como resultado de estos dos, se genera el amor a la marca donde se establecen pensamientos, sentimientos y experiencias agradables y memorables generando así una identificación, confianza y compromiso por la marca. 
COMPÁS EMPRESARIAL No 32

Vol. 11 - 1ER SEMESTRE 2021

ISSN: 2075-8960

Universidad Privada del Valle - Bolivia

https://doi.org/10.52428.20758960.v11i32.62

Avila Castañeda et al. (2018), adicionalmente, atribuyen que se puede mejorar esta relación cuando se define un género "masculino o femenino" a la marca. Machado et al., (2019) lograron establecer que el género juega un papel mediador entre el compromiso y el amor a la marca.

\section{c) Constructos de Intención de Compra}

La intención de compra se convierte en un factor clave a investigar, de sus resultados dependen el desarrollo de nuevos productos, las estimaciones de ventas, los canales de distribución a emplear y establecer el segmento de mercado, Ajzen, (1996) la define como la voluntad que el consumidor manifiesta en términos de esfuerzo y acción para realizar un determinado comportamiento, también se puede establecer como la predicción del comprador acerca de qué marca comprar, causando así un reflejo en el comportamiento de compra (Nasermoadeli et al., 2013).

De este modo, García (2014), con el fin de comprender el comportamiento del consumidor en el entorno electrónico, estableció que la intención de compra es la disposición que tiene el comprador instaurándose como variable dependiente, y como variables determinantes la confianza y valor percibido, formándose como el resultado de la evaluación sobre los beneficios funcionales y simbólicos al realizar la compra; aunque, en otro sentido, si la evaluación es negativa o si se percibe de la marca un problema ético o social afectará la confianza e intención de compra del consumidor (Lopes et al., 2020).

Por su parte, Singh \& Banerjee (2018) fundaron la relación de la credibilidad de las celebridades y de los anuncios publicitarios en la intención de compra, revelando que juegan un papel importante en la construcción de una marca positiva, pero destacan que para lograr una intención de compra, se debe establecer otros factores como la congruencia entre la marca y la celebridad; su posicionamiento; la relevancia del mensaje; la selección correcta del mercado objetivo y los medios de comunicación, junto con el atributo del producto al precio correcto, ya que la celebridad actúa, principalmente, como facilitador pera el desarrollo de un refuerzo positivo en la mente del consumidor. 
COMPÁS EMPRESARIAL No 32

Vol. 11 - 1ER SEMESTRE 2021

ISSN: 2075-8960

Universidad Privada del Valle - Bolivia

https://doi.org/10.52428.20758960.v11i32.62

Igualmente, Ugalde (2014) exploró la relación de la actitud y calidad percibida de una marca en la intención de compra, centrándose en el sector de servicios, estableciendo que la calidad percibida influye en la actitud hacia sus anuncios, pero que esta última no desencadena en la intención de compra. Por su parte, Slaba (2019), empleando la teoría del comportamiento planificado, establece tres factores: la experiencia, la sensibilidad al precio y lealtad, y cómo estos influyen en la intención de compra, propiamente de teléfonos móviles, revelando que el factor clave es la buena experiencia, permitiendo al consumidor una mejor dispocisión a pagar más por un producto o a recomendar a sus amigos.

Para finalizar, los avances tecnológicos y el crecimiento significativo de la demanda del mercado han llevado a la entrada acelerada de nuevos productos en el mercado, al tiempo que han generado un gran número de productos de desechos, es por esto por lo que Zhu, X., \& Yu, L. (2019) exploraron la intención de compra de productos remanufacturados, demostrando que la confianza y demanda de estos ha aumentado, permitiendo a las empresas mejorar sus procesos de desarrollo, aplicando modelos circulares de producción.

En tal sentido, Lee \& Chow (2020) examinan la influencia de las actitudes de los consumidores en sus intenciones para alquilar moda en línea, intentando entender cómo pueden participar en un consumo colaborarivo, siendo un proceso muy subjetivo para el consumidor, es por esto que las marcas o tiendas online deben de incentivar este tipo de acciones por medio de influenciadores o programas de incentivos, o de lealtad. De este último se puede decir que si se desarrollan de forma innovadora, generarán una respuesta favorable aumentando la lealtad de los consumidores, tal como lo presentan en su trabajo Hwang \& Choi (2020), quienes apoyan el papel escencial de los programas de fidelización gamificados en la gestión de las relaciones con los clientes, ofreciendo recompensas, mejorando la intención de los consumidores en la participación e involucramiento con la marca. 
COMPÁS EMPRESARIAL No 32

Vol. 11 - 1ER SEMESTRE 2021

ISSN: 2075-8960

Universidad Privada del Valle - Bolivia

https://doi.org/10.52428.20758960.v11i32.62

\section{CONCLUSIONES}

Como resultado de la revisión documental, podemos identificar una clara relación entre la confianza, compromiso y la intención de compra, las tres variables son interdependientes en el proceso de decisión de compra de los consumidores; desde el concepto de confianza podemos identificar la respuesta emocional y fiable cuando las marcas logran manifestar claramente los procesos de desarrollo de la propuesta de valor como las certificaciones, que puedan obtener un aval de calidad o sus propósitos sociales, pero a su vez, puede verse también afectada por las diferentes noticas o consecuencias negativas que se evidencien en diferentes medios o redes sociales.

Desde la revisión, del concepto de compromiso podemos evidenciar que cuando las marcas logran una relaciona profunda y sincera con el consumidor se generará de igual forma una respuesta positiva por parte del consumidor generando un comportamiento constante que al igual que la confianza es muy susceptible a la interacción que se pueda generar en contextos digitales o sociales.

Y, finalmente, las marcas para generar intención de compra de sus mercados objetivo no solamente deben desarrollar acciones dentro del marketing mix, sino que deben lograr integrar dentro de su proceso estratégico acciones para generar confianza y compromiso del consumidor, ya que se convierte en variables determinantes en el proceso de decisión de compra.

\section{REFERENCIAS}

Agyei, James, Shaorong Sun, Eugene Abrokwah, Emmanuel Kofi Penney, and Richmond OforiBoafo. 2020. "Influence of Trust on Customer Engagement: Empirical Evidence From the Insurance Industry in Ghana.” SAGE Open 10(1):215824401989910.

Ajzen, I. (1996). The directive influence of attitudes on behavior, in Gollwitzer, P.M., \& Bargh, J.A. (Ed.). The psychology of action: Linking cognition and motivation to behavior, (pp. 385-403). New York,USA: Guilford Press. 
COMPÁS EMPRESARIAL No 32

Vol. 11 - 1ER SEMESTRE 2021

ISSN: 2075-8960

Universidad Privada del Valle - Bolivia

https://doi.org/10.52428.20758960.v11i32.62

Avila Castañeda , A. L., Campos Pinzón, M. A., \& Chaparro Guevara, R. A. (2018). El Amor y el Amor por la Marca desde una Perspectica Conceptual. Santa Ana de Coro, Facón, Venezuela: Fondo Editorial Universitario Servando Garcés de la Universidad Politécnica Territorial.

Bianchi, Constanza, Valentina Reyes, and Veronica Devenin. 2020. "Consumer Motivations to Purchase from Benefit Corporations (B Corps)." Corporate Social Responsibility and Environmental Management (January):1-9.

Bonillo, M. (2002). ¿Qué entendemos por compromiso del consumidor? Estudios Sobre Consumo, ISSN 0212-9469, Nº 60, 2002, Pags. 71-88.

Boukes, Mark, Alyt Damstra, and Rens Vliegenthart. 2019. "Media Effects Across Time and Subject: How News Coverage Affects Two Out of Four Attributes of Consumer Confidence." Communication Research 009365021987008.

Busalim, Abdelsalam H., Ab Razak Che Hussin, and Noorminshah A. Iahad. 2019. "Factors Influencing Customer Engagement in Social Commerce Websites: A Systematic Literature Review.” Journal of Theoretical and Applied Electronic Commerce Research 14(2):0.

Calvo-Porral, C., Martínez-Fernández, V. A., \& Juanatey-Boga, S. (2013). Análisis de dos modelos de ecuaciones estructurales alternativos para medir la intencion de compra. Investigacion Operacional, 34(3), 230-243.

Chen, Ruixia, Zhimin Zhou, Ge Zhan, and Nan Zhou. 2020. "The Impact of Destination Brand Authenticity and Destination Brand Self-Congruence on Tourist Loyalty: The Mediating Role of Destination Brand Engagement.” Journal of Destination Marketing \& Management 15:100402.

Choi, \& Lee. (2019). Effect of Trust in Domain-Specific Information of Safety, Brand Loyalty, and Perceived Value for Cosmetics on Purchase Intentions in Mobile E-Commerce Context. Sustainability, 11(22), 6257. https://doi.org/10.3390/su11226257

Connell, Cara, Ruth Marciniak, Lindsey I. Carey, and Julie Mccoll. 2019. “Customer Engagement with Websites: A Transactional Retail Perspective.” European Journal of Marketing 53(9):1882-1904.

Dessart, Laurence, Joaquín Aldás-Manzano, and Cleopatra Veloutsou. 2019. "Unveiling Heterogeneous Engagement-Based Loyalty in Brand Communities.” European Journal of 
COMPÁS EMPRESARIAL No 32

Vol. 11 - 1ER SEMESTRE 2021

ISSN: 2075-8960

Universidad Privada del Valle - Bolivia

https://doi.org/10.52428.20758960.v11i32.62

Marketing 53(9):1854-81

Fedesarrollo. (30 de 05 de 2020). Fedesarrollo. Centro de investigación Económica y Social.

Obtenido de https://www.fedesarrollo.org.co/

Flavian, C., \& Guinalíu, M. (2006). La Confianza y el compromiso en las relaciones a través de Internet. Cuadernos de Economia Y Direccion de Empresa, 29, 133-160.

García, N. P. (2014). El valor percibido y la confianza como antecedentes de la intención de compra online: el caso colombiano. Cuadernos de Administración, 30(51), 15-24.

Garzaro, D. M., Varotto, L. F., Carvalho, M., \& Pedro, S. C. (2019). Interactivity and engagement: A systematic review of academic production in marketing. Revista Brasileira de Mrketing, 18(3), 246-265. https://doi.org/10.5585/remark.v18i3.16374

Gómez-Suárez, Mónica. 2019. "Examining Customer-Brand Relationships: A Critical Approach to Empirical Models on Brand Attachment, Love, and Engagement.” Administrative Sciences 9(1):10.

Hwang, J., \& Choi, L. (2020). Having fun while receiving rewards?: Exploration of gamification in loyalty programs for consumer loyalty. Journal of Business Research, 106(November 2017), 365-376. https://doi.org/10.1016/j.jbusres.2019.01.031

Jonkman, Jeroen, Mark Boukes, and Rens Vliegenthart. 2020. "When Do Media Matter Most? A Study on the Relationship between Negative Economic News and Consumer Confidence across the Twenty-Eight EU States.” The International Journal of Press/Politics 25(1):7695.

Kaur, Harsandaldeep, Mandakini Paruthi, Jamidul Islam, and Linda D. Hollebeek. 2020. "The Role of Brand Community Identification and Reward on Consumer Brand Engagement and Brand Loyalty in Virtual Brand Communities.” Telematics and Informatics 46:101321.

Larzelere, R., \& Huston, T. (1980). The Dyadic Trust Scale: Toward Understanding Interpersonal Trust in Close Relationships. Journal of Marriage and Family, 42(3), 595-604. doi: $10.2307 / 351903$

Lee, S. H. N., \& Chow, P.-S. (2020). Investigating consumer attitudes and intentions toward online fashion renting retailing. Journal of Retailing and Consumer Services, 52, 101892. https://doi.org/10.1016/j.jretconser.2019.101892

Li, Sai-Wei, Chen Zhu, Qi-Hui Chen, and Yu-Mei Liu. 2019. "Consumer Confidence and 
COMPÁS EMPRESARIAL No 32

Vol. 11 - 1ER SEMESTRE 2021

ISSN: $2075-8960$

Universidad Privada del Valle - Bolivia

https://doi.org/10.52428.20758960.v11i32.62

Consumers' Preferences for Infant Formulas in China.” Journal of Integrative Agriculture 18(8):1793-1803.

Lopes, E. L., Yunes, L. Z., Bandeira de Lamônica Freire, O., Herrero, E., \& Contreras Pinochet,

L. H. (2020). The role of ethical problems related to a brand in the purchasing decision process: An analysis of the moderating effect of complexity of purchase and mediation of perceived social risk. Journal of Retailing and Consumer Services, 53, 101970. https://doi.org/10.1016/j.jretconser.2019.101970

Machado, Joana César, Leonor Vacas-De-Carvalho, Salim L. Azar, Ana Raquel André, and Barbara Pires Dos Santos. 2019. "Brand Gender and Consumer-Based Brand Equity on

Facebook: The Mediating Role of Consumer-Brand Engagement and Brand Love." Journal of Business Research 96:376-85.

Marbach, Julia, Cristiana Lages, Daniel Nunan, and Yuksel Ekinci. 2019. "Consumer

Engagement in Online Brand Communities: The Moderating Role of Personal Values.”

European Journal of Marketing 53(9):1671-1700.

Nasermoadeli, A., Choon Ling, K., \& Maghnati, F. (2013). Evaluating the impacts of customer experience on purchase intention. International Journal of Business \& Management, 8 (6), 128-138.

Naumann, Kay, Jana Bowden, and Mark Gabbott. 2020. "Expanding Customer Engagement: The Role of Negative Engagement, Dual Valences and Contexts.” European Journal of Marketing ahead-of-p(ahead-of-print).

Ngo, Hai Minh, Ran Liu, Masahiro Moritaka, and Susumu Fukuda. 2020. "Urban Consumer

Trust in Safe Vegetables in Vietnam: The Role of Brand Trust and the Impact of Consumer Worry about Vegetable Safety.” Food Control 108(March 2019):106856.

Popova, Nadezhda, Andrei Kataiev, Ruslan Skrynkovskyy, and Anna Nevertii. 2019.

"Development of Trust Marketing in the Digital Society." Economic Annals-XXI 176(34):13-25.

Shawky, Sara, Krzysztof Kubacki, Timo Dietrich, and Scott Weaven. 2019. "Using Social Media to Create Engagement: A Social Marketing Review.” Journal of Social Marketing 9(2):20424. 
COMPÁS EMPRESARIAL No 32

Vol. 11 - 1ER SEMESTRE 2021

ISSN: 2075-8960

Universidad Privada del Valle - Bolivia

https://doi.org/10.52428.20758960.v11i32.62

Sheng, Jie. 2019. "Being Active in Online Communications: Firm Responsiveness and Customer

Engagement Behaviour.” Journal of Interactive Marketing 46:40-51.

Singh, R. P., \& Banerjee, N. (2018). Exploring the influence of celebrity credibility on brand attitude, advertisement attitude and purchase intention. Global Business Review, 19(6), 1622-1639. https://doi.org/10.1177/0972150918794974

Slaba, M. (2019). Consumer behaviour regarding branded and non-branded goods on the mobile phone market: impact of experience, price and loyalty. Economic Annals-XXI, 175(1-2), 63-67. https://doi.org/10.21003/ea.v175-1

Torres Valverde, E. P., \& Padilla Rivadeneira, G. S. (2013). Medición de la intención de compra con base en un modelo de regresión logística de productos de consumo masivo. 163. https://dspace.ups.edu.ec/bitstream/123456789/5772/1/UPS-QT03953.pdf

Ugalde, Cecilia. (2014). Influencia de la actitud hacia la marca y su calidad percibida en la actitud hacia sus anuncios: ¿afectan la intención de compra? Redmarka. Revista de Marketing Aplicado, 01(013), 43-62. https://doi.org/10.17979/redma.2014.01.013.487

Wang, Jianhua, Junying Tao, and May Chu. 2020. 'Behind the Label: Chinese Consumers' Trust in Food Certification and the Effect of Perceived Quality on Purchase Intention.” Food Control 108:106825.

Zhu, X., \& Yu, L. (2019). The Impact of Warranty Efficiency of Remanufactured Products on Production Decisions and Green Growth Performance in Closed-Loop Supply Chain: Perspective of Consumer Behavior. Sustainability, 11(5), 1420. https://doi.org/10.3390/su11051420

Zorio-Grima, Ana, and Paloma Merello. 2020. "Consumer Confidence: Causality Links with Subjective and Objective Information Sources.” Technological Forecasting and Social Change 150:119760.

Fuentes de financiamiento: Esta investigación fue financiada con fondos de los autores. Declaración de conflicto de intereses: Los autores declaran que no tienen ningún conflicto de interés. 
COMPÁS EMPRESARIAL No 32

Vol. 11 - 1ER SEMESTRE 2021

ISSN: 2075-8960

Universidad Privada del Valle - Bolivia

https://doi.org/10.52428.20758960.v11i32.62

Copyright (c) 2021 Ángel Leonardo Ávila Castañeda; Rosa Alexandra Chaparro Guevara

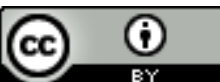

Este texto está protegido por una licencia $\underline{\text { CreativeCommons }} \underline{4.0}$.

Usted es libre para Compartir — copiar y redistribuir el material en cualquier medio o formato- y Adaptar el documento — remezclar, transformar y crear a partir del material - para cualquier propósito, incluso para fines comerciales, siempre que cumpla la condición de:

Atribución: Usted debe dar crédito a la obra original de manera adecuada, proporcionar un enlace a la licencia, e indicar si se han realizado cambios. Puede hacerlo en cualquier forma razonable, pero no de forma tal que sugiera que tiene el apoyo del licenciante o lo recibe por el uso que hace de la obra.

$\underline{\text { Resumendelicencia }-\underline{\text { Textocompletodelalicencia }}}$ 